\title{
Neuronale Korrelate unangenehmer somatosensorischer Stimulation bei Patienten mit Borderline-Persönlichkeitsstörung und Depression
}

Kathrin Malejko, Birgit Abler, Heiko Graf Klinik für Psychiatrie und Psychotherapie III, Universitätsklinikum Ulm

\section{ZUSAMMENFASSUNG}

Das Empfinden von unangenehmen Reizen, wie auch die Schmerzempfindung im engeren Sinne, wird nicht nur durch rein somatosensorische und nozizeptive Informationen, sondern ebenso durch affektive und kognitive Prozesse moduliert. Klinische Beobachtungen sowie wissenschaftliche Untersuchungen belegen eine veränderte Wahrnehmung der Schmerzempfindung bei psychischen Erkrankungen wie beispielsweise der emotional-instabilen Persönlichkeitsstörung vom Borderline-Typ (BPS) oder bei depressiven Störungen
(MD). Die Untersuchung der verschiedenen Modalitäten der Schmerzverarbeitung, welche zu einem großen Teil mit der Verarbeitung unangenehmer Reize überlappt, ist mittels verschiedener Stimulationsparadigmen möglich. Für die Darstellung der zugrunde liegenden neurofunktionellen Mechanismen eignet sich die funktionelle Magnetresonanztomografie (fMRT). Unter experimentellen Bedingungen kann der somatosensorisch-diskriminative Aspekt der Verarbeitung und Wahrnehmung von Schmerz und unangenehmen Reizen mit einem elektrischen Stimulationsparadigma operationalisiert werden. Die neuronalen Korrelate unangenehmer somatosensorischer Stimulation bei Patienten mit BPS und MD werden anhand eigener fMRT-Untersuchungen dargestellt und die veränderte Schmerzverarbeitung der beiden klinischen Gruppen erörtert.
Nach der Weltschmerzorganisation (IASP; International Association for the Study of Pain) ist Schmerz definiert als „ein unangenehmes Sinnes- und Gefühlserlebnis“, das mit einer „tatsächlichen oder drohenden Gewebeschädigung verknüpft ist oder mit Begriffen einer solchen beschrieben wird“ [1]. Operationalisiert man demnach Schmerz als unangenehmes Sinnes- und Gefühlserlebnis, so wird berücksichtigt, dass die Schmerzwahrnehmung nicht nur durch rein somatosensorische und nozizeptive Informationen, sondern ebenso durch affektive und kognitive Prozesse moduliert wird [2]. Klinische Beobachtungen sowie wissenschaftliche Untersuchungen weisen auf eine veränderte Schmerzwahrnehmung bei verschiedenen psychischen Erkrankungen wie der emotional-instabilen Persönlichkeitsstörung vom Borderline-Typ (BPS) [3-7] oder bei depressiven Störungen [8-13] hin. So war in Studien beispielsweise eine verminderte Schmerzempfindlichkeit $[8,10,11,13]$ und eine erhöhte Schmerzschwelle $[9,12]$ bei Patienten mit schwerer depressiver Episode (MD, major depression) zu beobachten. Patienten mit BPS berichten wiederum im Zusammenhang mit nicht suizidal selbstverletzendem Verhalten (NSSV) häufig von einer Hypalgesie [14]. Die zugrunde liegenden neurobiologischen Mechanismen des im Vergleich zu Gesunden veränderten Schmerzerlebens bei Patienten mit MD und BPS sind nur teilweise verstanden.

Um neurofunktionelle Prozesse der im Zusammenhang von Sinnes- und Gefühlserleben wie der Schmerzverarbeitung zu untersuchen, eignen sich funktionell bildgebende
Verfahren wie die funktionelle Magnetresonanztomografie (fMRT), welche die Darstellung der beteiligten neuronalen Strukturen ermöglichen. In diesem Zusammenhang wurde ein zunächst als „Schmerzmatrix“ $[15,16]$ bezeichnetes Netzwerk mit einer erhöhten neuronalen Aktivierung innerhalb der vorderen und hinteren Inselregion, des somatosensorischen Kortex, des Thalamus und des anterioren zingulären Kortex (ACC) bei der Applikation physischer Schmerzreize identifiziert. Während man anfänglich von einer schmerzspezifischen Aktivierung dieser Regionen ausging, zeigen neuere funktionell bildgebende Untersuchungen, dass dieses neuronale Netzwerk nicht nur rein nozizeptive, somatosensorische Verarbeitung abbildet, sondern verschiedene Modalitäten somatosensorischer Reize repräsentiert. So stehen neuronale Aktivierungen im primär und sekundär somatosensorischen Kortex (SI, SII), im Thalamus sowie in der hinteren Inselregion mit den sensorisch-diskriminativen Aspekten somatosensorischer Reize (z. B. Stimuluslokalisation, -dauer und -intensität) in Zusammenhang, während erhöhte Aktivierungen im ACC und in der vorderen Inselregion affektive, motivationale Aspekte neuronaler Verarbeitung somatosensorischer Reize repräsentieren [17, 18]. Neuronale Aktivierungen im präfrontalen Kortex (PFC), im dorsalen anterioren und midzingulären Kortex werden hingegen mit der kognitiven Bewertung von physischer Reize assoziiert [1921]. Die Untersuchung der Modalitäten der somatosensorischer Reizverarbeitung ist mittels verschiedener Stimulationsparadigmen möglich. 


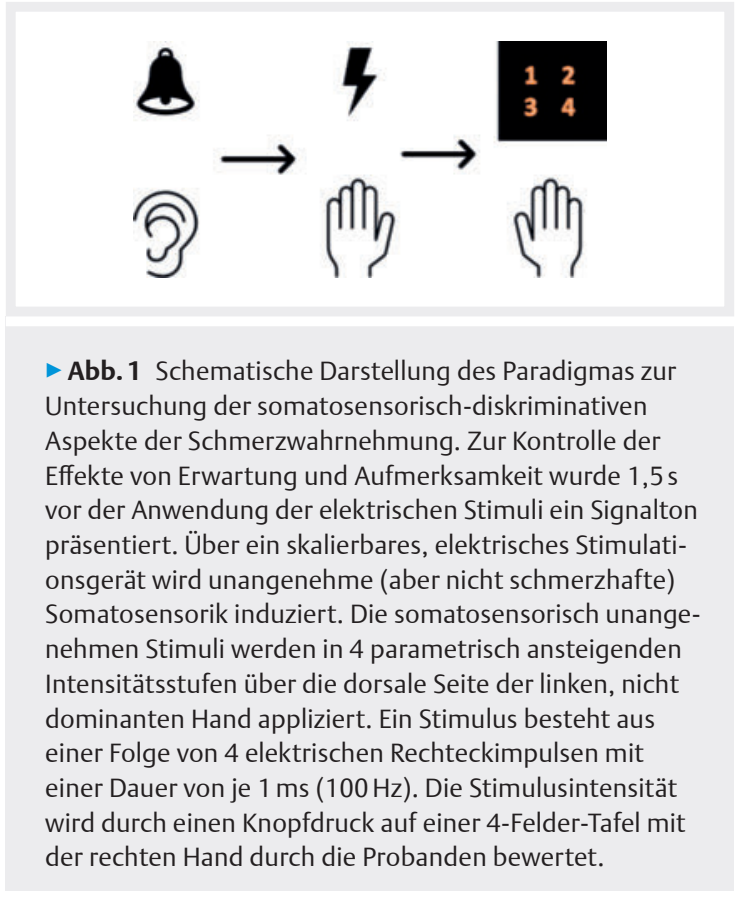

Zur Untersuchung somatosensorisch-diskriminativer Aspekte der Reizverarbeitung verwendeten wir eine Stimulationsmethode mit der sensorische Stimuli mit unangenehmem bis hin zu schmerzhaftem Charakter mittels elektrischer Stimulation an der Hautoberfläche appliziert werden ( Abb. 1) [7, 22-26]. Mittels fMRT lassen sich während der Stimulation mit unterschiedlichen, ansteigenden Stimulusintensitäten neurofunktionelle Korrelate der somatosensorischen Verarbeitung darstellen. Während der fMRT wird über ein elektrisches Stimulationsgerät ein nachweislich unangenehmer (jedoch nicht schmerzhafter) und in 4 parametrisch ansteigenden Intensitätsstufen skalierbarer somatosensorischer Reiz über die dorsale Seite der linken, nicht dominanten Hand appliziert. Ein Stimulus besteht aus einer Folge von 4 elektrischen Rechteckimpulsen mit einer Dauer von je $1 \mathrm{~ms}(100 \mathrm{~Hz})$. Die experimentellen Stimuli entsprechen den Richtlinien für experimentelle Schmerzen (nicht invasiv, keine Gewebeschädigung, Vermeidung von Bewegung, ethisch akzeptabel, reproduzierbar und physiologisch relevant) [27]. Die subjektiv empfundene Stimulationsintensität wird durch einen Knopfdruck auf einer 4-Felder-Tafel mit der rechten Hand durch die Studienteilnehmer angegeben. Um für Erwartungsund Aufmerksamkeitseffekte zu kontrollieren, wird etwa 1,5 s vor elektrischer Stimulation ein Signalton präsentiert.

Die elektrische Stimulation zur Applikation sensorisch unangenehm empfundener Reize und zur Untersuchung neurofunktioneller Repräsentationen der somatosensorisch-diskriminativen Komponente unangenehmer physischer Reize, wie sie auch Schmerzreize darstellen, erwies sich in einer fMRT-Untersuchung an gesunden Probanden als geeignet und reliabel. So waren neurofunktionelle Aktivierungen im primären und sekundären somatosenso- rischen Kortex und der hinteren Inselregion während der elektrischen Stimulation beobachtet worden [22]. Darüber hinaus zeigte sich eine mit ansteigender Reizintensität auch eine ansteigende neurofunktionelle Aktivierung [22]. Das Stimulationsparadigma sollte nun dazu dienen, möglichst störungsspezifische Unterschiede in neurofunktionellen Signaturen somatosensorisch-diskriminativer Reizverarbeitung bei Patienten mit psychischen Erkrankungen und insbesondere bei Patienten mit BPS und MD näher aufzuklären. Anhand der Darstellung eigener fMRT-Ergebnisse ist es das Ziel des Artikels, die Schmerzverarbeitung bei Patienten mit BPS und MD zu vermitteln. Betrachtet man Schmerz als Risikofaktor für mehrere psychische Erkrankungen [28, 29] und Störungen der Schmerzverarbeitung als ein diagnosenübergreifendes Symptom, so ist die Untersuchung neurofunktioneller Repräsentationen der Schmerzverarbeitung nicht nur von grundlagenwissenschaftlichem, sondern ebenso von klinischem Interesse.

\section{Emotional-instabile Persönlichkeits- störung vom Borderline-Typ}

Die BPS ist eine häufige Cluster-B-Persönlichkeitsstörung, die klinisch durch emotionale Instabilität, Störung des Sozialverhaltens und dysfunktionales Verhalten wie nicht suizidales selbstverletzendes Verhalten (NSSV) charakterisiert ist [30]. Häufig verletzen sich Betroffene dabei selbst durch Schnittwunden oder Verbrennungen. NSSV dient den Betroffenen dabei überwiegend der Affektregulation, mit dem eine aversiv erlebte innere Spannung reduziert werden kann [31-33]. Während des NSSVs berichten Patienten mit BPS wiederum häufig von einer Hypo- oder Analgesie [14]. Andererseits klagen Patienten mit BPS vermehrt über chronische und beeinträchtigende Schmerzen [34]. Eine im Vergleich zu Gesunden veränderte Schmerzwahrnehmung und -verarbeitung konnte entsprechend in mehreren Studien bei Patienten mit BPS beobachtet werden [4-6, 35-37].

In diesem Sinne zeigten mehrere Studien, welche mittels Hitze- und Kältereizen oder elektrischer Stimulation die Schmerzverarbeitung untersuchten, eine verminderte Schmerzempfindlichkeit bei Patienten mit BPS [3-7]. Bildgebende Untersuchungen konnten die bei Patienten mit BPS veränderte Schmerzwahrnehmung mit einer erhöhten neurofunktionellen Aktivierung im dorsolateralen präfrontalen Kortex (dIPFC) sowie einer verminderten neuronalen Aktivierung im vorderen ACC und in limbischen Regionen wie der Amygdala [4, 35, 36] in Verbindung bringen. Dieses im Vergleich zu Gesunden verändertes Aktivierungsmuster wurde als neurofunktionelles Korrelat eines antinozizeptiven Mechanismus interpretiert, in dem offenbar bei Patienten mit BPS insbesondere emotionale neurofunktionelle Komponenten der Schmerzverarbeitung durch eine verstärkte Top-Down-Regulation herunterreguliert werden. Ausgehend von der Vermutung einer veränderten affektiven 

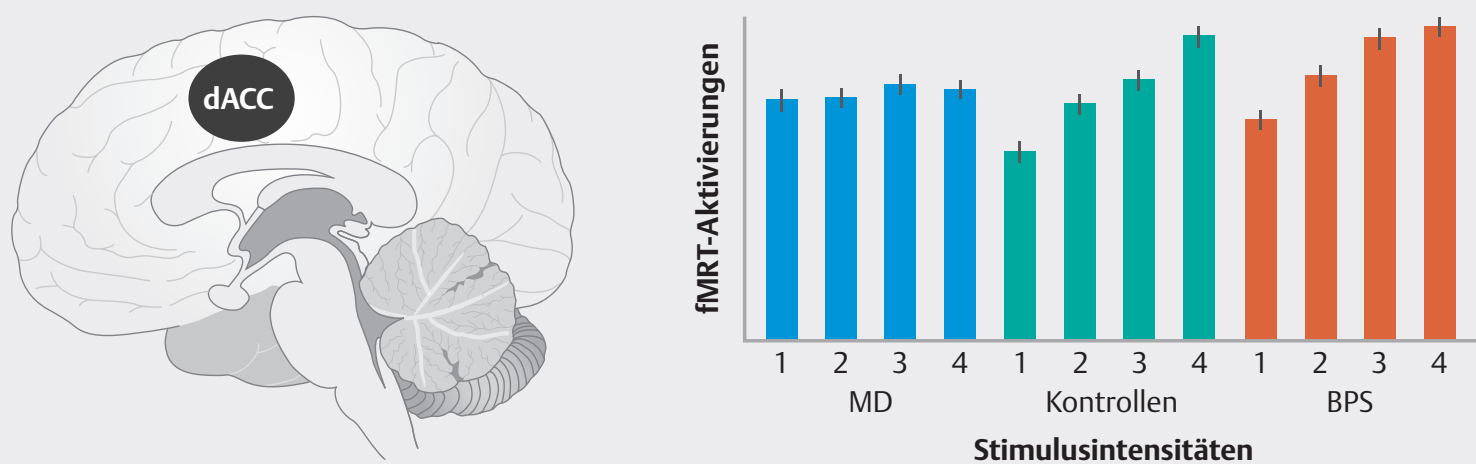

- Abb. 2 Die Abbildung zeigt keine absoluten Werte, sondern eine schematische Darstellung der fMRT-Ergebnisse während der sensorischen Stimulationsaufgabe mit parametrisch, ansteigenden Intensitäten (Stufen 1 bis 4) innerhalb des dorsalen anterioren zingulären Kortex (dACC) bei Patienten mit Borderline-Persönlichkeitsstörung (BPS), mit depressiver Störung (MD) und gesunden Kontrollprobanden. Das Balkendiagramm zeigt schematisch die fMRT-Aktivierung der Patienten mit BPS, MD und der gesunden Kontrollprobanden innerhalb des dACC (nach Daten aus $[23,25])$.

Schmerzbewertung, konzentrierten sich nachfolgende Studien vor allem auf die Interaktion von Schmerz und Affektregulation [36], sozialem Ausschluss [38] sowie auf zugrunde liegende Veränderungen der funktionellen Konnektivität bei BPS $[39,40]$. So konnte bspw. eine Studie bei Patienten mit BPS während des Schmerzempfindens durch Hitzereize eine verminderte funktionelle Konnektivität zwischen dem posteriorer zingulären Kortex (PCC) und dem dIPFC beobachten, die möglicherweise als neurofunktionelles Korrelat dafür anzusehen ist, dass die Betroffenen den Schmerz als weniger aversiv bewerten [39].

Die neurofunktionellen Repräsentationen rein somatosensorischer Aspekte von Schmerzwahrnehmung (z. B. Stimuluslokalisation, -dauer und -intensität) sind bei Patienten mit BPS jedoch kaum im Detail untersucht worden. Eine störungsspezifische Veränderung neurofunktionellen Korrelate insbesondere während der Wahrnehmung verschiedener Schmerzintensitäten war jedoch angesichts der reduzierten allgemeinen Schmerzempfindlichkeit bei Patienten mit BPS [3, 5, 6] und anhand des klinischen Eindrucks einer Hypalgesie im Zusammenhang mit selbstverletzendem Verhalten eine naheliegende Hypothese. In einer Studie [7] untersuchten wir 10 Patienten mit BPS im Vergleich zu Gesunden mit ansteigenden Reizintensitäten. Auf der Verhaltensebene zeigte sich kein signifikanter Unterschied hinsichtlich der subjektiven Einschätzung und der Diskriminationsfähigkeit der verschiedenen Intensitätsstufen zwischen Patienten mit BPS und gesunden Kontrollen. Zur Untersuchung der zugrunde liegenden neurofunktionellen Korrelate wurde das genannte Paradigma im nächsten Schritt während der fMRT bei 15 Patienten mit BPS und gesunden Probanden angewandt [23]. Während der Applikation elektrischer, unangenehmer Reize mit stufenweiser ansteigender Intensität konnten Patienten mit BPS und gesunde Kontrollprobanden die Intensitätsstufe der jeweiligen
Stimuli wiederum vergleichbar gut einschätzen. Bei Patienten mit BPS waren jedoch etwas höhere Stimulusintensitäten erforderlich, damit diese als vergleichbar unangenehm empfunden worden waren. Übereinstimmend zu der im Vergleich zu Gesunden unveränderten Wahrnehmung unterschiedlicher Schmerzintensitäten, zeigte sich auch während der fMRT-Untersuchung eine neurofunktionelle Aktivierung im SI, SII, in der hinteren und mittleren Inselregion sowie im midzingulären Kortex, die in Abhängigkeit von der jeweiligen Stimulusintensität sowohl bei Patienten mit BPS und Gesunden zunahm. Unterschiede in den neurofunktionellen Aktivierungen zwischen den Gruppen (BPS und Gesunde) waren jedoch nicht zu beobachten ( $\triangleright$ Abb. 2). Zusammenfassend kann daher angenommen werden, dass sich die sensorisch-diskriminative Komponente der Schmerzwahrnehmung weder auf der Verhaltensebene noch hinsichtlich der neurofunktionellen Repräsentation bei Patienten mit BPS im Vergleich zu Gesunden unterscheidet [23]. Diese Ergebnisse stützen die Annahme, dass die im Vergleich zu Gesunden veränderte Schmerzwahrnehmung bei Patienten mit BPS überwiegend aus einer veränderten emotionalen, kognitiven Bewertung resultiert. Sensorisch-diskriminative Vorgänge und korrespondierende neurofunktionelle Repräsentationen der Schmerzwahrnehmung unterscheiden sich bei Patienten mit BPS hingegen nicht von denen bei Gesunden. Übereinstimmend hierzu zeigte sich in einer Untersuchung [7], dass bei Gesunden mit ansteigender Reizintensität an unangenehmen sensorischen Stimuli auch ein ansteigendes Erregungsniveau und innere Anspannung einherging, während dieser Anstieg emotionaler Aspekte bei Patienten mit BPS nicht zu beobachten war. Weiter konnte die Arbeitsgruppe von Schmahl et al. [41] zeigen, dass Stress ein starker Modulator des Schmerzempfindens bei der BPS darstellt und dazu führt, dass Patienten mit BPS eine Hyp- oder Analgesie trotz Induktion von Schmerzstimuli hoher Intensitäten (z. B. während NSSV durch Schneiden) verspüren, wogegen die senso- 
risch-diskriminativen Aspekte der Schmerzverarbeitung unbeeinträchtigt bleiben.

\section{Depressive Störung}

Klinische Beobachtungen und zahlreiche Untersuchungen lassen einen Zusammenhang zwischen dem Empfinden von Schmerzen und depressiven Störungen vermuten. Patienten mit MD klagen häufig über multiple Schmerzen [42] und es zeigte sich umgekehrt eine erhöhte Prävalenz an depressiven Störungen bei Patienten mit chronischen Schmerzerkrankungen [43]. Das Interesse an der grundlagenwissenschaftlichen Aufklärung der Schmerzverarbeitung bei MD nahm demnach in den letzten Jahren deutlich zu [9-11, 44-47]. Neuere Studien zu grundlegenden Mechanismen der Wahrnehmung von Schmerz oder von unangenehmen Reizen [9-11, 44-48] zeigen wiederum, dass Patienten mit MD im Vergleich zu Gesunden häufig eine verminderte physische Schmerzempfindlichkeit aufweisen $[8,10,11,13]$. So wurden erhöhte Reizschwellen bei schmerzhaften bzw. unangenehmen Stimuli beobachtet, wenn Druck, thermische oder elektrische Reize auf die Haut ausgeübt wurden [9, 12]. Die Wahrnehmung von Kälte und Wärme unterschied sich wiederum bei Patienten mit MD nicht von der bei Gesunden [49]. Möglicherweise spielen veränderte Abläufe bei entzündlichen Prozessen und Veränderungen in der Regulation der Hypothalamus-Hypophysen-Nebennierenrinde-Achse (HPA) bei der Interaktion zwischen Schmerz und Depression eine Rolle [50, 51]. So beobachteten Untersuchungen am Tiermodell bei chronischem Stress eine erhöhte Zytokinkonzentration in den Raphe-Kernen, im Thalamus, Hippocampus, im präfrontalen Kortex und in der Hypophyse und ein depressives Verhalten der Tiere sowie eine Hyperalgesie [50, 51].

Bislang stehen nur wenige bildgebende Untersuchungen zur Verfügung, die neurofunktionelle Repräsentationen der Schmerzwahrnehmung und -verarbeitung unangenehmer somatosensorischer Stimuli bei Patienten mit MD untersuchten, und diese zeigten zudem uneinheitliche Befunde: Eine fMRT-Studie zu neuronalen Aktivierungen während eines leicht schmerzhaften $45^{\circ} \mathrm{C}$-Wärmereizes bei erwachsenen Patienten mit MD zeigte eine erhöhte Schmerzschwelle sowie eine relative Überaktivierung im präfrontalen Kortex während der Schmerzapplikation im Vergleich zu Gesunden [44]. Strigo et al. beobachteten eine erhöhte neurofunktionelle Aktivierung in der vorderen Inselregion, in der rechten Amygdala und im hinteren ACC während der Erwartung schmerzhafter Wärmereize bei jungen Erwachsenen mit depressiver Episode als mögliche neurofunktionelle Repräsentation von Angst bzw. einer erhöhten affektiven Beteiligung vor dem Erleben des Reizes bei MD [45, 46]. Im Gegensatz hierzu waren wiederum neurofunktionelle Aktivierungen im vorderen ACC und im präfrontalen Kortex während des Schmerzerlebens vermindert [46]. Der dorsale ACC und die Inselregion sind als Teil des Salienz-Netzwerkes für die neurofunktionelle Verarbeitung auffallender, herausragender Stimuli verantwortlich. Übereinstimmend hierzu zeigten weitere Untersuchungen bei Patienten mit MD ebenfalls eine veränderte neuronale Reaktion im dorsalen ACC und in der Inselregion während negativer, nicht notwendigerweise schmerzhafter Stimuli, und damit in Regionen des Salienz-Netzwerks [52, 53]. Die bei Patienten mit MD beobachtete veränderte Schmerzverarbeitung könnte demnach auch mit einer erhöhten neurofunktionellen Aktivierung im Salienz-Netzwerk zusammenhängen.

Basierend auf diesen Ergebnissen einer veränderten neurofunktionellen Verarbeitung von schmerzhaften Reizen bei Patienten mit MD untersuchten wir, ob Veränderungen innerhalb des neuronalen Netzwerks, das die neurofunktionelle Verarbeitung unangenehmer sensorischer Reize repräsentiert, auch bei nicht schmerzhafter sensorischer Stimulation beobachtbar sind. Um diese Annahmen näher aufzuklären, untersuchten wir 22 Patienten mit MD im Vergleich zu 25 Gesunden mit fMRT während der Stimulation mit parametrisch ansteigenden und zunehmend unangenehmen elektrischen Stimuli [25]. Dabei zeigte sich bei Patienten mit MD im Vergleich zu Gesunden eine nahezu aufgehobene Modulation neurofunktioneller Aktivierungen, unabhängig von der zunehmenden Intensität an unangenehmen elektrischen Reizen ( $\triangleright$ Abb. 2). Diese ausbleibende Modulation neurofunktioneller Aktivierungen zeigte sich dabei in Gehirnarealen, die sowohl an der somatosensorischen als auch an der affektiven Verarbeitung von physischem Schmerz, unangenehmen sensorischen Reizen und salienten Stimuli beteiligt sind. Weiterhin bemerkenswert ist jedoch, dass Patienten mit MD subjektiv die verschiedenen, zunehmenden Intensitäten an unangenehmen Stimuli auch als zunehmend intensiv und unangenehm diskriminieren konnten, während korrespondieren neurofunktionelle Aktivierungen diesen parametrischen Anstieg nicht widerspiegelten. Darüber hinaus waren bei Patienten mit MD bereits vergleichsweise niedrige Intensitäten an unangenehmen elektrischen Reizen ausreichend, um ein zu Gesunden vergleichbares subjektives Empfinden des unangenehmen Reizes sowie eine vergleichbar hohe neurofunktionelle Aktivierung zu provozieren. Die Ergebnisse dieser Studie deuten demnach auf eine deutlich abweichende neurofunktionelle Repräsentation unangenehmer sensorischer Empfindungen bei der MD hin, die möglicherweise durch eine verstärkte Wahrnehmung negativer Stimuli, aber auch durch eine verminderte Aktivierung und Modulation neurofunktioneller Korrelate somatosensorisch-diskriminativer Reize erklärt werden kann. Möglicherweise kann dieser Befund zur Erklärung der bei depressiven Patienten beobachteten veränderten Schmerzwahrnehmung beitragen. 
FAZIT

Das Empfinden von unangenehmen Reizen wird, wie auch die Schmerzempfindung im engeren Sinne, sowohl durch rein somatosensorisch-diskriminative, aber auch durch emotionale und kognitive Komponenten moduliert. Wenngleich die Aufklärung der neurofunktionellen Repräsentationen dieser Komponenten im Fokus wissenschaftlicher Untersuchungen stand, so waren mögliche Veränderungen dieser neurofunktionellen Signaturen bei Patienten mit psychischen Störungen noch weitestgehend ungeklärt. Die Aufklärung neurofunktioneller Mechanismen der Wahrnehmung sensorisch unangenehmer Reize bei psychischen Störungen ist dabei nicht nur von grundlagenwissenschaftlichem Interesse, sondern insbesondere motiviert durch die klinische Beobachtung einer im Vergleich zu Gesunden veränderten Schmerzwahrnehmung. So berichten beispielsweise Patienten mit BPS von einer An- oder Hypalgesie während nicht suizidalem selbstverletzendem Verhalten, und Patienten mit BPS und MD vermehrt von chronischen Schmerzen. Bislang konzentrierten sich neurowissenschaftliche Untersuchungen vorwiegend auf affektive oder kognitive Aspekte der Schmerzverarbeitung bei Patienten mit BPS und MD. Mit fMRT untersuchten wir ein Kollektiv an Patienten mit BPS sowie Patienten mit MD im Vergleich zu Gesunden und fokussierten auf somatosensorisch-diskriminative Aspekte der Schmerzwahrnehmung und die zugrunde liegenden neurofunktionellen Mechanismen.

Während parametrisch ansteigender, elektrischer Stimulation zur Untersuchung der sensorisch-diskriminativen Komponente unangenehmer Reize zeigte sich bei Patienten mit BPS im Vergleich zu Gesunden keine Beeinträchtigung hinsichtlich der subjektiven Diskrimination der verschiedenen, ansteigenden Intensitäten unangenehmer sensorischer Reize. Übereinstimmend hierzu zeigte sich sowohl bei Patienten mit BPS als auch bei Gesunden ein parametrischer Anstieg an neurofunktionellen Aktivierungen, die mit somatosensorisch-diskriminativen Aspekten der Schmerzverarbeitung assoziiert sind. Demnach kann davon ausgegangen werden, dass die veränderte Schmerzwahrnehmung akuter Schmerzen im Rahmen von Selbstverletzungen bei Patienten mit BPS in einer veränderten kognitiven, emotionalen Bewertung begründet ist, und unsere Ergebnisse unterstützen damit die Befunde früherer Untersuchungen. Bei Patienten mit MD konnte im Vergleich zu Gesunden hingegen eine fehlende Modulation neurofunktioneller sensorisch-diskriminativer Komponenten beobachtet werden, die sich mit parametrisch ansteigender Stimulusintensität nicht erhöhten. Auf der Verhaltensebene zeigte sich wiederum eine im Vergleich zu Gesunden unbeeinträchtigte subjektive Bewertung der Stimulusintensitäten. Berücksichtigt man die bei Patienten mit MD fehlende neurofunktionelle Modulation in zerebralen Regionen, die sowohl an der somatosensorischen Schmerzverarbeitung, als auch an der Verarbeitung salienter Stimuli beteiligt ist, so kann dies dahingehend interpretiert werden, dass bei depressiven Störungen eine veränderte subjektive Wahrnehmung unangenehmer Reize, zu denen auch Schmerzen gehören, mit einer verstärkten Wahrnehmung negativer Stimuli, aber auch mit einer veränderten sensorisch-diskriminativen Komponente erklärt werden kann.

Interessenkonflikt

Erklärung zu finanziellen Interessen

Forschungsförderung erhalten: nein; Honorar/geldwerten Vorteil für Referententätigkeit erhalten: nein; Bezahlter Berater/interner Schulungsreferent/Gehaltsempfänger: nein; Patent/Geschäftsanteile/Aktien (Autor/Partner, Ehepartner, Kinder) an Firma (Nicht-Sponsor der Veranstaltung): nein; Patent/Geschäftsanteile/Aktien (Autor/Partner, Ehepartner, Kinder) an Firma (Sponsor der Veranstaltung): nein.

Erklärung zu nicht finanziellen Interessen

Die Autoren geben an, dass kein Interessenkonflikt besteht.

\section{Korrespondenzadresse}

\author{
Dr. med. Kathrin Malejko \\ Universitätsklinik Ulm \\ Klinik für Psychiatrie und Psychotherapie III \\ Leimgrubenweg 12-14, 89075 Ulm \\ Deutschland \\ Tel. 0731/50061558, Fax 0731/50061402 \\ kathrin.malejko@uni-ulm.de
}

\section{Literatur}

[1] Classification of chronic pain. Descriptions of chronic pain syndromes and definitions of pain terms. Prepared by the International Association for the Study of Pain, Subcommittee on Taxonomy. Pain Suppl 1986; 3: S1-226. https://pubmed. ncbi.nlm.nih.gov/3461421/

[2] Peters ML. Emotional and Cognitive Influences on Pain Experience. Mod Trends Pharmacopsychiatry 2015; 30: 138-152

[3] Bohus M, Limberger M, Ebner $\mathrm{U}$ et al. Pain perception during self-reported distress and calmness in patients with 
borderline personality disorder and self-mutilating behavior. Psychiatry Res 2000; 95: 251-260

[4] Schmahl C, Bohus M, Esposito F et al. Neural correlates of antinociception in borderline personality disorder. Arch Gen Psychiatry 2006; 63: 659-667

[5] Ludascher P, Bohus M, Lieb K et al. Elevated pain thresholds correlate with dissociation and aversive arousal in patients with borderline personality disorder. Psychiatry Res 2007; 149: 291-296

[6] Ludascher P, Bohus M, Lieb K et al. Elevated pain thresholds correlate with dissociation and aversive arousal in patients with borderline personality disorder. Psychiatry Res 2007; 149: 291-296. doi:10.1016/j.psychres.2005.04.009

[7] Schmahl C, Greffrath W, Baumgartner U et al. Differential nociceptive deficits in patients with borderline personality disorder and self-injurious behavior: laser-evoked potentials, spatial discrimination of noxious stimuli, and pain ratings. Pain 2004; 110: 470-479. doi:10.1016/j.pain.2004.04.035

[8] Cardenas-Morales L, Fladung AK, Kammer T et al. Exploring the affective component of pain perception during aversive stimulation in borderline personality disorder. Psychiatry Res 2011; 186: 458-460. doi:10.1016/j.psychres.2010.07.050

[9] Adler G, Gattaz WF. Pain perception threshold in major depression. Biol Psychiatry 1993; 34: 687-689. doi:10.1016/0006-3223(93)90041-b

[10] Bar KJ, Brehm S, Boettger MK et al. Pain perception in major depression depends on pain modality. Pain 2005; 117 : 97-103. doi:10.1016/j.pain.2005.05.016

[11] Bar KJ, Greiner W, Letsch A et al. Influence of gender and hemispheric lateralization on heat pain perception in major depression. J Psychiatr Res 2003; 37: 345-353. doi:10.1016/ s0022-3956(03)00051-7

[12] Schwier C, Kliem A, Boettger MK et al. Increased cold-pain thresholds in major depression. J Pain 2010; 11: 287-290. doi:10.1016/j.jpain.2009.07.012

[13] Lautenbacher S, Spernal J, Schreiber W et al. Relationship between clinical pain complaints and pain sensitivity in patients with depression and panic disorder. Psychosom Med 1999; 61: 822-827. doi:10.1097/00006842-19991100000015

[14] Dickens C, McGowan L, Dale S. Impact of depression on experimental pain perception: a systematic review of the literature with meta-analysis. Psychosom Med 2003; 65: 369-375. doi:10.1097/01.psy.0000041622.69462.06

[15] Leibenluft E, Gardner DL, Cowdry RW. The inner experience of the borderline self-mutilator. Journal of Personality Disorders 1987; 1: 317-324. doi:10.1521/pedi.1987.1.4.317

[16] Wager TD, Atlas LY, Lindquist MA et al. An fMRI-based neurologic signature of physical pain. N Engl J Med 2013; 368: 1388-1397. doi:10.1056/NEJMoa 1204471

[17] Rainville P, Duncan GH, Price DD et al. Pain affect encoded in human anterior cingulate but not somatosensory cortex. Science 1997; 277: 968-971. doi:10.1126/science. 277.5328 .968

[18] Peyron R, Laurent B, Garcia-Larrea L. Functional imaging of brain responses to pain. A review and meta-analysis (2000). Neurophysiol Clin 2000; 30: 263-288. doi:10.1016/s09877053(00)00227-6

[19] Davis KD. The neural circuitry of pain as explored with functional MRI. Neurol Res 2000; 22: 313-317. doi:10.1080/016 16412.2000 .11740676
[20] Tan LL, Pelzer P, Heinl C et al. A pathway from midcingulate cortex to posterior insula gates nociceptive hypersensitivity. Nat Neurosci 2017; 20: 1591-1601. doi:10.1038/nn.4645

[21] Buchel C, Bornhovd K, Quante M et al. Dissociable neural responses related to pain intensity, stimulus intensity, and stimulus awareness within the anterior cingulate cortex: a parametric single-trial laser functional magnetic resonance imaging study. J Neurosci 2002; 22: 970-976

[22] Vogt BA. Midcingulate cortex: Structure, connections, homologies, functions and diseases. J Chem Neuroanat 2016; 74: 28-46. doi:10.1016/j.jchemneu.2016.01.010

[23] Adolph O, Koster S, Georgieff M et al. Xenon-induced changes in CNS sensitization to pain. Neuroimage 2010; 49: 720-730. doi:10.1016/j.neuroimage.2009.08.034

[24] Malejko K, Neff D, Brown RC et al. Somatosensory Stimulus Intensity Encoding in Borderline Personality Disorder. Front Psychol 2018; 9: 1853. doi:10.3389/fpsyg.2018.01853

[25] Bonenberger M, Plener PL, Groschwitz RC et al. Polymorphism in the micro-opioid receptor gene (OPRM1) modulates neural processing of physical pain, social rejection and error processing. Exp Brain Res 2015; 233: 2517-2526. doi:10.1007/s00221-015-4322-9

[26] Malejko K, Brown RC, Plener PL et al. Differential neural processing of unpleasant sensory stimulation in patients with major depression. Eur Arch Psychiatry Clin Neurosci 2020. doi:10.1007/s00406-020-01123-0

[27] Bonenberger M, Plener PL, Groschwitz RC et al. Differential neural processing of unpleasant haptic sensations in somatic and affective partitions of the insula in non-suicidal self-injury (NSSI). Psychiatry Res 2015; 234: 298-304. doi:10.1016/j. pscychresns.2015.10.013

[28] Petersen-Felix S, Arendt-Nielsen L. From pain research to pain treatment: the role of human experimental pain models. Best Pract Res Clin Anaesthesiol 2002; 16: 667-680. doi:10.1053/bean.2002.0258

[29] Bair M], Robinson RL, Katon W et al. Depression and pain comorbidity: a literature review. Arch Intern Med 2003; 163 : 2433-2445

[30] Breivik H, Collett B, Ventafridda $V$ et al. Survey of chronic pain in Europe: prevalence, impact on daily life, and treatment. Eur J Pain 2006; 10: 287-333

[31] Al-Alem L, Omar HA. Borderline personality disorder: an overview of history, diagnosis and treatment in adolescents. Int J Adolesc Med Health 2008; 20: 395-404

[32] Kleindienst N, Bohus M, Ludascher P et al. Motives for nonsuicidal self-injury among women with borderline personality disorder. J Nerv Ment Dis 2008; 196: 230-236. doi:10.1097/NMD.0b013e3181663026

[33] Shearer SL. Phenomenology of self-injury among inpatient women with borderline personality disorder. J Nerv Ment Dis 1994; 182: 524-526

[34] Willis F, Kuniss S, Kleindienst $N$ et al. Stress reactivity and pain-mediated stress regulation in remitted patients with borderline personality disorder. Brain Behav 2018; 8: e00909. doi:10.1002/brb3.909

[35] Biskin RS, Frankenburg FR, Fitzmaurice GM et al. Pain in patients with borderline personality disorder. Personal Ment Health 2014; 8: 218-227. doi:10.1002/pmh.1265

[36] Niedtfeld I, Schmitt R, Winter D et al. Pain-mediated affect regulation is reduced after dialectical behavior therapy in borderline personality disorder: a longitudinal fMRI study. Soc Cogn Affect Neurosci 2017; 12: 739-747. doi:10.1093/ scan/nsw183 
[37] Niedtfeld I, Schulze L, Kirsch P et al. Affect regulation and pain in borderline personality disorder: a possible link to the understanding of self-injury. Biol Psychiatry 2010; 68: 383-391. doi:10.1016/j.biopsych.2010.04.015

[38] Schmahl C, Bremner JD. Neuroimaging in borderline personality disorder. J Psychiatr Res 2006; 40: 419-427. doi:10.1016/j.jpsychires.2005.08.011

[39] Bungert M, Koppe G, Niedtfeld I et al. Pain Processing after Social Exclusion and Its Relation to Rejection Sensitivity in Borderline Personality Disorder. PLoS One 2015; 10: e0133693. doi:10.1371/journal.pone.0133693

[40] Kluetsch RC, Schmahl C, Niedtfeld I et al. Alterations in default mode network connectivity during pain processing in borderline personality disorder. Arch Gen Psychiatry 2012; 69: 993-1002. doi:10.1001/archgenpsychiatry.2012.476

[41] Niedtfeld I, Kirsch P, Schulze L et al. Functional connectivity of pain-mediated affect regulation in Borderline Personality Disorder. PLoS One 2012; 7: e33293. doi:10.1371/journal. pone.0033293

[42] Schmahl C, Baumgartner U. Pain in Borderline Personality Disorder. Mod Trends Pharmacopsychiatry 2015; 30: 166-175. doi:10.1159/000435940

[43] Vaccarino AL, Sills TL, Evans KR et al. Multiple pain complaints in patients with major depressive disorder. Psychosom Med 2009; 71: 159-162. doi:10.1097| PSY.0b013e3181906572

[44] Ohayon MM, Schatzberg AF. Chronic pain and major depressive disorder in the general population. I Psychiatr Res 2010; 44: 454-461. doi:10.1016/j.jpsychires.2009.10.013

[45] Bar KJ, Wagner G, Koschke M et al. Increased prefrontal activation during pain perception in major depression. Biol Psychiatry 2007; 62: 1281-1287. doi:10.1016/j.biopsych.2007.02.011

[46] Strigo IA, Matthews SC, Simmons AN. Decreased frontal regulation during pain anticipation in unmedicated subjects with major depressive disorder. Transl Psychiatry 2013; 3: e239. doi:10.1038/tp.2013.15

[47] Strigo IA, Simmons AN, Matthews SC et al. Association of major depressive disorder with altered functional brain response during anticipation and processing of heat pain. Arch Gen Psychiatry 2008; 65: 1275-1284. doi:10.1001/ archpsyc.65.11.1275
[48] Rodriguez-Raecke R, Ihle K, Ritter C et al. Neuronal differences between chronic low back pain and depression regarding long-term habituation to pain. Eur J Pain 2014; 18: 701-711. doi:10.1002/j.1532-2149.2013.00407.x

[49] Han C, Pae CU. Pain and depression: a neurobiological perspective of their relationship. Psychiatry Investig 2015; 12 : 1-8. doi:10.4306/pi.2015.12.1.1

[50] Klauenberg S, Maier C, Assion H] et al. Depression and changed pain perception: hints for a central disinhibition mechanism. Pain 2008; 140: 332-343. doi:10.1016/j. pain.2008.09.003

[51] Zhao J, Gao X, Wang A et al. Depression comorbid with hyperalgesia: Different roles of neuroinflammation induced by chronic stress and hypercortisolism. J Affect Disord 2019; 256: 117-124. doi:10.1016/j.jad.2019.05.065

[52] Norman G], Karelina K, Zhang $N$ et al. Stress and IL-1beta contribute to the development of depressive-like behavior following peripheral nerve injury. Mol Psychiatry 2010; 15 : 404-414. doi:10.1038/mp.2009.91

[53] Hamilton JP, Etkin A, Furman DJ et al. Functional neuroimaging of major depressive disorder: a meta-analysis and new integration of base line activation and neural response data. Am J Psychiatry 2012; 169: 693-703. doi:10.1176/appi. ajp.2012.11071105

[54] Manoliu A, Meng C, Brandl F et al. Insular dysfunction within the salience network is associated with severity of symptoms and aberrant inter-network connectivity in major depressive disorder. Front Hum Neurosci 2013; 7: 930. doi:10.3389/ fnhum.2013.00930

Bibliografie

DOI https://doi.org/10.1055/a-1298-6508

Nervenheilkunde 2021; 40: 53-59

(C) 2021. Thieme. All rights reserved.

Georg Thieme Verlag KG, Rüdigerstraße 14,

70469 Stuttgart, Germany

ISSN 0722-1541 


\section{Punkte sammeln auf CME.thieme.de}

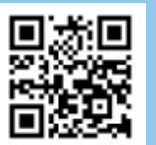

Diese Fortbildungseinheit ist bis zu 12 Monate online für die Teilnahme verfügbar.

Den genauen Einsendeschluss finden Sie beim Modul auf https://cme.thieme.de/CXGZG28.

Sollten Sie Fragen zur Online-Teilnahme haben, finden Sie unter https://cme.thieme.de/hilfe

eine ausführliche Anleitung. Wir wünschen viel Erfolg beim Beantworten

der Fragen!

Unter https://eref.thieme.de/CXGZG28 oder über den QR-Code kommen Sie

direkt zum Artikel.

VNR 2760512021160213637

\section{Frage 1}

Schmerzwahrnehmung wird in der Regel operationalisiert als ...

A die kognitive Dimension einer nozizeptiven Information.

B das Vorhandensein eines unangenehmen physikalischen Reizes.

C ein unangenehmes Sinneserleben, welches affektiv oder kognitiv beeinflusst sein kann.

D die Stimulation von C-Fasern und A-delta-Fasern.

E eine tatsächliche oder drohende Gewebeschädigung.

\section{Frage 2}

Welche Aussage zur Schmerzwahrnehmung und selbstverletzendem Verhalten ist falsch?

A Selbstverletzendes Verhalten kann suizidal intendiert sein.

B Selbstverletzendes Verhalten kann nicht suizidal intendiert sein.

C Patienten mit einer schwergradigen Depression haben oft eine erhöhte Schmerzschwelle.

D Bei Patienten mit Borderline-Persönlichkeitsstörung beobachtet man oft eine Hyperalgesie im Zusammenhang mit selbstverletzendem Verhalten.

E Patienten mit Borderline-Persönlichkeitsstörung haben oft eine veränderte Schmerzwahrnehmung.

\section{Frage 3}

Welche Aussage zu Patienten mit depressiver Störung (major depression, MD) ist richtig?

A Patienten mit MD haben das gleiche Schmerzempfinden wie Gesunde.

B Patienten mit MD sind bei der Wahrnehmung von Wärme und Kälte empfindlicher als Gesunde.

C Patienten mit MD sind lärmempfindlicher als Gesunde.

D Patienten mit MD haben eine verminderte Reizschwelle auf physikalische somatosensorische Reize.

E Patienten mit MD sind weniger empfindlich auf schmerzhafte oder unangenehme somatosensorische Stimuli.

\section{Frage 4}

Welche Aussage zur Schmerzmatrix ist falsch?

A Der rechte Gyrus angularis ist der Hauptknotenpunkt des Netzwerkes.

B Das Netzwerk repräsentiert verschiedene Modalitäten somatosensorischer Reize.

C Der somatosensorische Kortex und der Thalamus sind Bestandteile der Schmerzmatrix.

D Die vordere und hintere Inselregion sowie der anteriore zinguläre Kortex sind Bestandteile der Schmerzmatrix.

E Es lassen sich sensorisch-diskriminative Aspekte von affektiven und motivationealen Aspekten neuronaler Verarbeitung sensorischer Reize unterscheiden.

\section{Frage 5}

Zu dem sogenannten Salienz-Netzwerk im Gehirn gehören:

A ventrales ACC und insulärer Kortex.

B Insel und dorsales ACC.

C frontales Augenfeld und rechter insulärer Kortex.

D dorsaler parietaler Kortex und Putamen.

E Amygdala und Präkuneus.

\section{Frage 6}

Welche Aussage zu nicht suizidalem selbstverletzendem Verhalten (NSSV) bei Patienten mit Borderline-Persönlichkeitsstörung (BPS) ist falsch?

A Bei Patienten mit BPS tritt häufig NSSV auf.

B NSSV wird bei Patienten mit BPS oft zur Affektregulation eingesetzt.

C Oft führen Patienten mit BPS NSSV in Form von Ausreißen von Finger- oder Fußnägeln durch.

D Während NSSV schildern Patienten mit BPS häufig eine Hypo- oder Analgesie.

E Mit NSSV versuchen Patienten mit BPS oft, eine unangenehme innere Spannung zu reduzieren. 


\section{Punkte sammeln auf CME.thieme.de}

Fortsetzung $\ldots$

\section{Frage 7}

Welche Aussage zu den Ergebnissen einer experimentellen Studie mit fMRT zur Schmerzwahrnehmung bei Patienten mit BPS und gesunden Kontrollen ist richtig?

A Die veränderte Schmerzwahrnehmung von Patienten mit BPS beruht überwiegend auf einer Alteration der kognitiven und emotionalen Bewertung der Schmerzreize.

B Bei Patienten mit BPS findet sich keine abgestufte neurofunktionelle Aktivierung bei Erhöhung der Stimulationsintensität.

C Bei gesunden Probanden nimmt die Aktivierung im somatosensorischen Kortex und der hinteren und mittleren Inselregion mit zunehmender Stimulationsintensität ab.

D Die sensorisch-diskriminative Repräsentation der Schmerzwahrnehmung von Patienten mit BPS unterscheidet sich deutlich von der Repräsentation gesunder Kontrollpersonen.

E Während die Schmerzwahrnehmung bei Gesunden den somatosensorischen Kortex, die hintere und mittlere Inselregion sowie den zingulären Kortex aktiviert, zeigt sich bei Patienten mit BPS überwiegend eine Aktivierung des linken und rechten dorsolateralen präfrontalen Kortex.

\section{Frage 8}

Welche Aussage ist falsch? In einem Schmerzexperiment mit Patienten mit depressiver Störung unter funktioneller Bildgebung ...

A unterschieden sich die Patienten von den gesunden Kontrollpersonen bezüglich der Modulation auf parametrisch ansteigende elektrische Stimuli.

B fand sich bei den Patienten eine andere neurofunktionelle Repräsentation unangenehmer Reize als bei den untersuchten Gesunden.

C wurde das subjektive Empfinden eines unangenehmen Reizes bei Patienten mit höheren Intensitäten erzielt als bei gesunden Probanden.

D zeigten sich Unterschiede zwischen Patienten und Gesunden in somatosensorischen Arealen und Bereich der affektiven Verarbeitung von Schmerz.

E bestand bei Patienten eine Diskrepanz zwischen der Diskriminierbarkeit von Schmerzreizen und der korrespondierenden neurofunktionellen Aktivierung.

\section{Frage 9}

Welche Aussage zu chronischem Schmerz ist richtig?

A Nur Patienten mit BPS, nicht jedoch Patienten mit MD, berichten vermehrt von chronischem Schmerz.

B Nur Patienten mit MD, nicht jedoch Patienten mit BPS, berichten vermehrt von chronischem Schmerz.

C Weder Patienten mit MD noch Patienten mit BPS berichten von chronischem Schmerz.

D Sowohl Patienten mit MD als auch Patienten mit BPS berichten von chronischem Schmerz.

E Bei keiner psychiatrischen Erkrankung leiden die Patienten vermehrt unter chronischem Schmerz.

\section{Frage 10}

Welche Aussage zur veränderten Schmerzwahrnehmung bei Patienten mit MD in dem hier dargestellten Schmerzexperiment ist falsch?

A Es zeigt sich eine fehlende neurofunktionelle Modulation in den aktivierten zerebralen Regionen.

B Die aktivierten zerebralen Regionen sind an der somatosensorischen Verarbeitung von Schmerz beteiligt.

C Eine veränderte subjektive Wahrnehmung unangenehmer Reize, zu denen auch Schmerzen gehören, kann mit einer mit veränderten sensorisch-diskriminativen Komponente der Schmerzverarbeitung erklärt werden.

D Die aktivierten zerebralen Regionen sind an der Verarbeitung salienter Stimuli beteiligt.

E Eine veränderte subjektive Wahrnehmung unangenehmer Reize, zu denen auch Schmerzen gehören, kann mit einer verstärkten Wahrnehmung positiver Stimuli erklärt werden. 